\title{
Catecholaminergic Polymorphic Ventricular Tachycardia by ECG Finding
}

National Cancer Institute

\section{Source}

National Cancer Institute. Catecholaminergic Polymorphic Ventricular Tachycardia by

ECG Finding. NCI Thesaurus. Code C99936.

An electrocardiographic finding of ventricular tachycardia that is associated with syncope and/or cardiac arrest triggered by emotion or exercise in patients whose baseline ECG is normal. (ACC) 